\title{
Application of plant growth simulation algorithm for permutation flow shop scheduling problem
}

\author{
Baohua Yan ${ }^{1, a}$ \\ ${ }^{1}$ Gansu Normal University for Nationalities, Hezuo, gansu, 747000, China \\ aBaohuayan@163.com
}

Keywords: Plant Growth Simulation Algorithm; Permutation flow shop scheduling problem; intelligent algorithms; global optimum problem

\begin{abstract}
Based on Plant Growth Simulation Algorithm, we propose a novel algorithm for solving permutation flow shop scheduling problems. Starting with the characteristic of permutation flow shop scheduling problems, combining disjunctive graph according to the sequence of scheduling, analyzing Scheduling options that decided by exchangeable set and alternative set. Simulation results that the Plant Growth Simulation Algorithm (PGSA) has better feasibility and validity for solving permutation flow shop scheduling problem.
\end{abstract}

\section{Introduction}

The permutation flow shop scheduling problem has been widely studied in the recent decades. An flow shop system consists of $m$ machines in a serial layout where a set of $n$ jobs has to go through first machine one, then machine two, and so on until machine $\mathrm{m}$. That is, all the jobs have identical routes. Each job requires an operation with uninterrupted processing time on each of the machines. Each job can be preceded at most one machine at the same time. The processing of a job on a machine cannot be interrupted. All jobs are independent and are available for processing at time 0; and etc. The aim is to find a sequence for processing all jobs on all machines so that the given criterion is minimized. Although the process constraint of the model is relatively simple, but it has proven more than 3 machine of permutation flow shop scheduling problem is NP problem ${ }^{[1]}$.

More attention was paid on applying intelligent bionic algorithm to solve the problem in recently decades. Evolutionary algorithms such as simulated annealing algorithm ${ }^{[2]}$, ant colony algorithm ${ }^{[3]}$, PSO algorithm ${ }^{[4]}$ etc. Evolutionary algorithm can improve the population size and the number of iterations to improve accuracy, the solving time is proportional to iterative times, time efficiency decreased obviously.Some scholars, which based on the simulation the behavior of swarm intelligence, presented for solving combinatorial optimization problems, Such as genetic algorithms, particle swarm optimization, ant colony algorithm, swarm algorithm and this paper will discuss the simulation of plant growth algorithm. Those intelligent algorithms have been widely used in many areas, also include job shop production scheduling problem ${ }^{[5-7]}$.

The paper proposes a new effective hybrid algorithm is proposed which is based on plant growth simulation algorithm for solving the permutation flow shop scheduling problem, simulation example verifies the correctness and effectiveness of the algorithm.

\section{Mathematical description of permutation flow shop scheduling problem}

Permutation flow shop scheduling problem studies the flow process of $\mathrm{n}$ work pieces on $\mathrm{m}$ machines. If the scheduling objective is the maximum completion time, the case of Permutation flow shop scheduling problem mathematics is described as follows: $n$ represents the number of jobs, $m$ represents the machine number, prmu shows that all the workpiece after each machine processing consistency, $C_{\max }$ represents the maximum completion time of the work pieces, $t_{i j}$ denotes the processing time of workpiece $i$ on machine $j, C\left(j_{i}, k\right)$ represents the completion time of workpiece $j_{i}$ on machine $k, \pi$ represents a sort of all jobs, $T$ is collection of all sort. Assume that the workpieces 
are processed according to machine 1 to $\mathrm{m}$, then the completion time of $n$ work pieces on $m$ machines can be obtained by formula(1) to formula(5) .

$$
\begin{aligned}
& c\left(j_{1}, 1\right)=t_{j_{1}, i} \\
& c\left(j_{i}, 1\right)=c\left(j_{i-1}, 1\right)+t_{j_{i}, i} i=2 \ldots . n \\
& c\left(j_{1}, k\right)=c\left(j_{1}, k-1\right)+t_{j_{1}, k} k=2 \ldots . m \\
& c\left(j_{i}, k\right)=\max \left\{c\left(j_{i}, k-1\right), c\left(j_{i-1}, k\right)\right\}+t_{j_{i}, k} i=2 \ldots n, k=2 \ldots . m \\
& c_{\max }(\pi)=c\left(j_{n}, m\right) \\
& \pi^{\prime}=\arg \left\{c_{\max }(\pi)=c\left(j_{n}, m\right)\right\} \rightarrow \min , \forall \pi \in T
\end{aligned}
$$

Which formula (5) is the maximum completion time, formula (6) represents the corresponding scheduling scheme of minimize the maximum completion time.

\section{The plant growth simulation algorithm}

The plant growth simulation algorithm is a bionicrandom algorithm ${ }^{[8]}$. It looks at the feasible region of integer programming as the growth environment of a plant and determines the probabilities to grow a new branch on different nodes of a plant according to the change of the objective function, and then makes the model, which simulates the growth process of a plant, rapidly growth towards the light source (global optimum solution).

In the growth process, in order to get enough sunlight for photosynthesis, the plants strive to breed more branches and leaves. This character of plants is called phototropism. There are two facts for the growth law of plants.

A.The higher the morphactin concentration of a node, the greater the probabilty to grow a new branch on the node;

B. The morphactin concentration of any node on a plant is not given beforahand and is not fixed; however, it is determined by the environmental information of the node which depends on its relative position on the plant. The morphactin concentrations of all nodes of a plant are allotted again according to the new environment information after it grows a new branch.

It was proved by biological experiments that a new branch that is able to grow depends on its morphactin concentration when the plant has more than one node. The node which has larger value of morpheme concentration has more growth opportunities than the smaller one, morphactin concentration are not pre-assigned to the nodes, but formed according to their location information which we call that plant showed the feature of plant's Phototropism. After the node has been formed, morphactin concentration will be readjusted according to changing in the environment of a new growth node else.

We could describe the characteristics of plant growth in term of the mathematical view basis on plant growth analysis of the above. Suppose the length of tree trunk is T, the length of branch is $\mathrm{L}$, there are $\mathrm{W}$ growing nodes $S_{T}=\left(S_{T 1}, S_{T 2}, \ldots, S_{T W}\right)$ in the trunk, morphactin concentration of those nodes is $P_{T}=\left(P_{T 1}, P_{T 2}, \ldots, P_{T W}\right)$ respectively. There are $q$ growth nodes $S_{T}=\left(S_{T 1}, S_{T 2}, \ldots, S_{T q}\right)$ in the branch. Morphactin concentration of those nodes is $P_{T}=\left(P_{T 1}, P_{T 2}, \ldots, P q\right)$ respectively. The morphactin concentration which grown in the trunk and branch is calculated as follows:

$$
P_{T i}=f\left(x_{0}\right)-f\left(S_{T i}\right) / \sum_{i}^{W}\left(f\left(x_{0}\right)-f\left(S_{T i}\right)\right)+\sum_{j}^{q}\left(f\left(x_{0}\right)-f\left(x_{T q}\right)\right)
$$

Where $x_{0}$ represents the root of plant (the initial node), $f(*)$ is the information function of the node in the environment, The smaller value of the function as possible shows the better environment where the node located, it will help to grow new branch.

From formula (7), the value of morphactin concentration in every node depends on relative position of the relative to the initial root and the location of the environmental information, the 
mechanism consistent with the formation of the morphactin concentration in the plant cell. We can deduced from formula (7) as follows:

$$
\sum_{i=1}^{T} \sum_{j=1}^{q}\left(P_{T i}+P_{T j}\right)=1
$$

We can produce random number in the interval $[0,1]$. The random number must fall within one of the state space $\left(P_{1}, P_{2}, \ldots, P_{T+q}\right)$, where the corresponding node will have a prior right to grow new branch. A simple example of morphactin concentration state space displays in the fig. 1.The value of morphactin concentration in all nodes will change after the new branch has grown up. Computation formula of the value of morphactin concentration in other nodes will add the relevant node on the new branch, and remove the grown node, which basis on formula (7). The process will be repeated until there is no new branches growing.

\section{The analysis of simulation}

In order to explain the performance of the proposed algorithm, the numerical examples used in this paper are generated by random method. Examples are 10 Liao benchmark problems ${ }^{[9]}$. Each example contains 5 stages. The number of parallel machines in each phase is distributed between 3 and 5 . The range of the workpiece processing time is $[1,1000]$. On this basis, the assumption that all the machines are not always available,they may have a failure, the failure of the machine will be immediately repaired, where the time of machine failure and repair spent are subject to normal distribution. In the experiment, there are 5 failures in the setting of the machine, and the mean value of fault time is 200, 400, 600, 800 and 1000 respectively. The mean value of the time used in the repair is 20 and the variance is 5. Run environment of algorithm is MATLAB (R2010b) under Win7 operating system. Under two situations of the algorithm in preempt-resume (case 1) and preempt-repeat (case 2) solving results respectively as shown in Table 1 and 2.

Table 1 Comparison results on benchmark problems of Casel

\begin{tabular}{|c|l|l|l|l|l|l|l|l|l|l|l|l|}
\hline $\begin{array}{c}\text { Pro } \\
\text { blem }\end{array}$ & \multicolumn{4}{|c|}{ PSO } & \multicolumn{2}{c|}{ RKGA } & \multicolumn{3}{c|}{ IA } & \multicolumn{3}{c|}{ PGSA } \\
\cline { 2 - 13 } & AVE & MIN & SD & AVE & MIN & SD & AVE & MIN & SD & AVE & MIN & SD \\
\hline j30c5el & 511.7 & 507 & 2.4 & 513.0 & 505 & 3.9 & 509.0 & 506 & 1.5 & 503.1 & 501 & 1.5 \\
\hline j30c5e2 & 670.3 & 670 & 0.4 & 670.5 & 670 & 0.8 & 670.0 & 670 & 0.0 & 670.0 & 670 & 0.0 \\
\hline j30c5e3 & 663.6 & 657 & 3.7 & 667.5 & 655 & 7.5 & 659.6 & 655 & 1.7 & 654.0 & 651 & 1.5 \\
\hline j30c5e4 & 631.3 & 628 & 2.1 & 630.7 & 626 & 3.1 & 629.2 & 627 & 1.2 & 622.2 & 621 & 1.1 \\
\hline j30c5e5 & 668.5 & 666 & 1.7 & 671.7 & 666 & 4.5 & 662.8 & 662 & 0.4 & 662.6 & 662 & 0.4 \\
\hline j30c5e6 & 675.4 & 670 & 3.3 & 679.0 & 670 & 5.6 & 669.1 & 665 & 2.5 & 663.5 & 661 & 2.2 \\
\hline j30c5e7 & 686.1 & 684 & 1.8 & 686.0 & 683 & 2.5 & 683.9 & 682 & 0.9 & 681.1 & 681 & 0.4 \\
\hline j30c5e8 & 737.9 & 732 & 3.2 & 738.3 & 731 & 5.1 & 734.2 & 731 & 1.8 & 729.8 & 729 & 1.1 \\
\hline j30c5e9 & 708.8 & 705 & 2.0 & 709.8 & 703 & 5.1 & 705.4 & 702 & 1.6 & 699.6 & 698 & 1.2 \\
\hline j30c5el0 & 650.5 & 641 & 5.3 & 648.8 & 636 & 7.2 & 642.5 & 637 & 2.2 & 634.9 & 631 & 1.9 \\
\hline Average & 660.4 & 656 & 2.6 & 661.5 & 655 & 4.5 & 656.6 & 654 & 1.4 & 652.1 & 651 & 1.1 \\
\hline
\end{tabular}


Table 2 Comparison results on benchmark problems of Case2

\begin{tabular}{|l|l|l|l|l|l|l|l|l|l|l|l|l|}
\hline \multirow{2}{*}{ Problem } & \multicolumn{3}{|c}{ PSO } & \multicolumn{3}{c|}{ RKGA } & \multicolumn{3}{c|}{ IA } \\
\cline { 2 - 14 } & AVE & MIN & SD & AVE & MIN & SD & AVE & MIN & SD & AVE & MIN & SD \\
\hline j30c5el & 561.6 & 557 & 5.1 & 562.1 & 555 & 8.7 & 559.6 & 554 & 2.4 & 552.7 & 549 & 2.7 \\
\hline j30c5e2 & 720.0 & 701 & 4.4 & 723.8 & 701 & 7.8 & 716.3 & 701 & 3.2 & 700.7 & 697 & 2.7 \\
\hline j30c5e3 & 747.5 & 739 & 6.0 & 748.6 & 735 & 6.2 & 743.0 & 736 & 4.5 & 723.3 & 712 & 4.6 \\
\hline j30c5e4 & 702.2 & 696 & 7.5 & 701.3 & 694 & 8.1 & 697.5 & 688 & 5.9 & 685.3 & 674 & 5.9 \\
\hline j30c5e5 & 733.7 & 726 & 8.7 & 734.1 & 726 & 13. & 731.5 & 726 & 5.9 & 725.1 & 709 & 3.8 \\
\hline j30c5e6 & 737.1 & 730 & 3.6 & 737.0 & 720 & 6.0 & 730.7 & 718 & 3.1 & 718.1 & 708 & 8.8 \\
\hline j30c5e7 & 752.2 & 745 & 4.3 & 751.8 & 743 & 6.9 & 747.9 & 741 & 3.8 & 732.1 & 723 & 4.7 \\
\hline j30c5e8 & 836.7 & 802 & 10. & 839.2 & 792 & 14. & 826.4 & 790 & 9.5 & 786.8 & 771 & 6.7 \\
\hline j30c5e9 & 799.4 & 791 & 6.8 & 800.2 & 789 & $\begin{array}{l}15 . \\
4\end{array}$ & 793.6 & 788 & 5.9 & 766.9 & 760 & 4.8 \\
\hline j30c5el0 & 748.9 & 740 & 3.2 & 746.2 & 715 & 11. & 738.1 & 712 & 9.3 & 718.5 & 710 & 8.7 \\
\hline Average & 733.9 & 723 & 6.0 & 734.4 & 717 & 9.9 & 728.5 & 715 & 5.4 & 710.9 & 701 & 5.3 \\
\hline
\end{tabular}

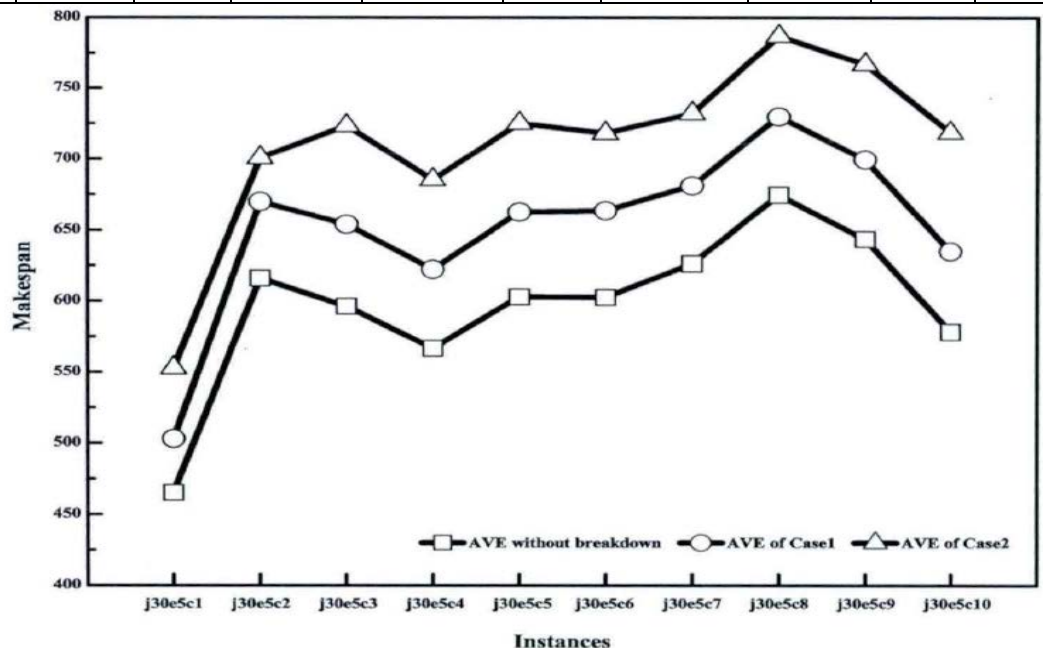

Fig1. The computational results under two breakdown cases and no breakdown case

From the test data can be seen, for the selected Liao problems, the plant growth simulation algorithm has good optimization performance in the field of combinatorial optimization, and it is an effective tool for solving the permutation flow shop scheduling problem.

\section{Conclusions}

This paper proposes an improve bat algorithm, the improve plant growth simulation algorithm(PGSA) is applied to solve the permutation flow shop scheduling problem, the performance has been improved obviously, and as the problem size increases, the improvement is more obvious. Experiments show that: The algorithm is effective and robust, it is feasible and effective for solving the permutation flow shop scheduling problem. It shows a good application prospect in production scheduling field.

\section{References}

[1] Garey M R,Johnson D S,Sethi R. The complexity of floe shop and job shop scheduling[J]. Mathematics of operations research, 1976,1(2):117-129.

[2] Low C, Yeh J Y, Huang K I. A robust simulated annealing heuristic for flow shop scheduling problems[J]. International Journal of Advanced Manufacturing Technology,2004,23:762-767. 
[3] Liu Yanfeng, Liu Sanyang. An Ant Colony Optimization for permutation flow shop scheduling[J]. Systems engineering and electronics, 2008, 30(9):1690-1692.

[4] Liu B, Wang L, Jin Y H. An effective hybrid PSO-based algorithm for flow shop scheduling with limited buffers[J]. Computers \& operations research, 2008, 35(9):2791-2806.

[5] Pinedo M.Scheduling: theory, algorithms, and systems, 2rd Prentice-Hall, Englewood Cliffs, New Jersey. 2002,20(4): 126-144.

[6] Rao Y Q, Yan Z X, et al. Application of a Hybrid Genetic Algorithm for Job Shop Scheduling [J].Mechanical Science and Technology, 2006, 25 (5):584-587,607.

[7] Liu Y, Ye C M, Shen Y H. Solving the Job shop scheduling problem based on improved particle swarm algorithm [J]. Systems Engineering and Electronics, 2006, 28 (4):602-606.

[8] Tasgetiren M F, Liang G Y, Sevkli M,et al. A particle swarm optimization algorithm for make span and total flow time minimization in the permutation flow shop sequencing problem[J]. European Journal of operational research,2007,177:1930-1947.

[9] Liao C. J., Tjandradjaja E., Chung T. P. An approach using particle swarm optimization and bottleneck heuristic to solve hybrid flow shop scheduling problem[J]. Applied Soft Computing, 2012, 12(6): 1755-1764. 